\title{
Combining Ability and Type of Gene Action Analysis of Yield and Yield Components for Some White Maize Inbred Lines
}

\author{
H. E. Mosa; ia.i. El-gazzar and m.a.a. Hassan \\ Maize research department, fcri, arc, Egypt \\ Corresponding author: mosasayed@yahoo.com
}

\begin{abstract}
Combining ability analysis were conducted for grain yield, its components, plant and ear heights and days to $50 \%$ silking in a half diallel cross involving eight white maize inbred lines. The resulting 28 hybrids and two commercial hybrids (SC10 and SC128) were grown at Sakha, Gemmeiza and Mallawy Research Stations in 2014 growing season. Both general and specific combining ability and their interaction with locations were significantly for most traits. However, the additive gene effects was most responsible for controlling the inheritance of ear height, ear length, ear diameter, number of rows/ear and number of kernels/row while the non additive gene effects was higher than additive gene effects for days to $50 \%$ silking, plant height and grain yield. Also, the non-additive gene effects was more interacted with locations than additive gene effects for most studied traits. The best general combiner inbred line was $\mathrm{P}_{3}$ for earliness, $\mathrm{P}_{8}$ for short plant and ear heights, $\mathrm{P}_{5}$ for ear length, number of kernels/row and grain yield, $\mathrm{P}_{7}$ for ear diameter and $\mathrm{P}_{1}$ for number of rows/ear. The best hybrid for specific combining ability was $\mathrm{P} 4 \mathrm{x} \mathrm{P}_{7}$ for grain yield.
\end{abstract}

Keywords: Maize, combining ability, additive gene effects, non-additive gene effects.

\section{Introduction}

The conventional crop breeding methodology mainly depends upon the development of inbred lines of maize from open pollinated varieties or other heterogeneous sources and their evaluation through different techniques to evolve desirable hybrids for commercial use. Diallel crosses have been used in genetic research to determinate the inheritance of a trait among a set of genotypes to identify superior parents for hybrids development (Yan and Hunt, 2003). The variance of general combining ability GCA and specific combining ability SCA are related to the type of gene action involved. Variance for GCA includes additive portion while that of SCA includes non-additive portion of total variance arising largely from dominance and epistatic deviations (Rojas and Sprague, 1952), superiority of a line on the basis of combining ability estimates can only be decided precisely after knowing the purpose of a certain breeding program whether, it is to develop high yielding open pollinated varieties or the superior combination of hybrids. Lines which had higher GCA effects can be used in synthetic variety development more effectively. However, when high yield specific combination are desired, especially in hybrid maize development, SCA effects could help in the selection parental material for hybridization.

The objectives of this study were to estimate the genetic parameters and to determine suitable parents and promising crosses for eight traits in $8 \times 8$ half diallel crosses.

\section{Materials and Methods}

Eight white inbred lines, $\mathrm{P}_{1}$ (Sk-6001/6), $\mathrm{P}_{2}$ (Sk-6005/8), $\mathrm{P}_{3}$ (Sk-5001/65), P4 (Sk-5002/36), $\mathrm{P}_{5}$ (Sd-34), $\mathrm{P}_{6}$ (Sk-8), $\mathrm{P}_{7}$ (Sk-12) and $\mathrm{P}_{8}$ (Sk-13) obtained from the Maize Research Department, Field Crops Research Institute, (FCRI), Agricultural Research Center (ARC), Egypt were crossed in a $8 \mathrm{x}$ 8 half diallel mating scheme in the 2013 growing season. The resulting $28 \quad \mathrm{~F}_{1}$ hybrids and two commercial hybrids SC10 and SC128 were grown at three Agricultural Research Stations, Sakha, Gemmeiza and Mallawy in 2014 growing season. The plots were represented by 1 row, while the row to row and plant to plant distances were kept $80 \mathrm{~cm}$ and $25 \mathrm{~cm}$, respectively, with 25 plants per row after thinning. The experiment design was a randomized complete block design with 4 replications. All agronomic and cultural practices were applied as recommended in the three locations. Data were recorded for days to $50 \%$ silking, plant and ear heights $(\mathrm{cm})$, ear length and diameter $(\mathrm{cm})$, number of rows/ear, number of kernels/row and grain yield (ard/fed) adjusted based $15.5 \%$ grain moisture (one $\operatorname{ardab}=140 \mathrm{~kg}$ and one feddan $=4200 \mathrm{~m}^{2}$ ). Combined analysis across three locations for 30 hybrids was carried out when homogeneity of variance was detected, which locations were considered as random effects and hybrids were considered as fixed effects (Steel and Torrei, 1980) and further the 28 hybrids were analysed for combining ability using method-4, model-1 (Griffing, 1956).

\section{Results and Discussion}

Combined analysis of variance for eight traits across three locations are presented in Table (1). 
Differences among three locations (L) were found to be highly significant for all studied traits, indicating markedly differences between the three locations in their climate and soil conditions.

Table 1. Combined analysis of variance for eight traits across three locations.

\begin{tabular}{|c|c|c|c|c|c|c|c|c|c|}
\hline SOV & $\mathrm{df}$ & $\begin{array}{l}\text { Days to } \\
50 \% \\
\text { silking }\end{array}$ & $\begin{array}{l}\text { Plant } \\
\text { Height }\end{array}$ & $\begin{array}{l}\text { Ear } \\
\text { height }\end{array}$ & Ear length & $\begin{array}{l}\text { Ear } \\
\text { diameter }\end{array}$ & $\begin{array}{l}\text { No. of } \\
\text { rows/ear }\end{array}$ & $\begin{array}{l}\text { No. of } \\
\text { kernels/ } \\
\text { row }\end{array}$ & $\begin{array}{l}\text { Grain } \\
\text { yield }\end{array}$ \\
\hline $\begin{array}{l}\text { Locations } \\
\text { (Loc) }\end{array}$ & 2 & $554.919 * *$ & $91166.336^{* *}$ & $38581.96^{* *}$ & $936.36 * *$ & $7.921 * *$ & $172.672 * *$ & $4.394 .84 * *$ & $3601.06 * *$ \\
\hline Rep./Loc & 9 & 3.535 & 470.517 & 223.07 & 5.956 & 0.138 & 2.492 & 112.52 & 27.07 \\
\hline Hybrids (H) & 27 & $17.626^{* *}$ & $1172.865^{* *}$ & $555.339 * *$ & $18.809 * *$ & $0.258^{*}$ & $7.548 * *$ & $69.373 * *$ & $105.39 * *$ \\
\hline Checks (C) & 1 & 45.37 & 2035.04 & 2185.04 & 0.327 & 0.54 & 1.5 & 2.67 & 2.73 \\
\hline H vs. C & 1 & 22.27 & 1742.06 & 161.39 & 0.05 & 0.26 & 22.81 & 0.898 & $232.42 *$ \\
\hline $\mathrm{H} \times \mathrm{L}$ & 54 & $3.197 * *$ & $301.645^{* *}$ & $232.7 * *$ & $3.72 * *$ & $0.156^{*}$ & $1.959 * *$ & $21.82 *$ & $45.9 * *$ \\
\hline $\mathrm{Ch} \times \mathrm{L}$ & 2 & $3.375 *$ & $378.3^{*}$ & $735.79 * *$ & 2.49 & 0.24 & 0.62 & 2.73 & 3.65 \\
\hline H vs. Ch x L & 2 & $11.22 * *$ & $363.199 *$ & 171.924 & 5.44 & 0.077 & 2.95 & $54.07 *$ & 3.96 \\
\hline Error & 261 & 0.830 & 118.743 & 79.59 & 1.860 & 0.083 & 0.951 & 13.15 & 10.38 \\
\hline
\end{tabular}

Significant at 0.05 and 0.01 levels of probability, respectively

In Table 2. Sakha location produced high means for eight traits while the reverse was obtained, at Mallawy location. Frey (1964) defined the stress environment as one in which mean performance for certain attribute is low. Regarding to Table 1, the differences among the hybrids were significant or highly significant for all studied traits while the differences among check (C) and hybrids vs checks
(H vs C) were not significant for all traits except $(\mathrm{H}$ vs C) for grain yield. Also, the $\mathrm{H} x \mathrm{~L}$ interaction was significant or highly significant for all traits, while $\mathrm{C}$ $\mathrm{x} \mathrm{L}$ and $\mathrm{H}$ vs $\mathrm{C} \times \mathrm{L}$ were not significant for most traits. Comstoc and Moll (1963) defined the genotypes $\mathrm{x}$ environments, interaction as the differential response of genotypes to the change in environments.

Table 2. Means of three locations for eight traits.

\begin{tabular}{lllllllll}
\hline Location & $\begin{array}{l}\text { Days to } \\
50 \% \text { silking height }(\mathrm{cm})\end{array}$ & $\begin{array}{l}\text { Plant } \\
(\mathrm{cm})\end{array}$ & $\begin{array}{l}\text { Ear } \\
(\mathrm{cm})\end{array}$ & $\begin{array}{l}\text { Ear } \\
\text { diameter } \\
(\mathrm{cm})\end{array}$ & $\begin{array}{l}\text { No. of } \\
\text { rows/ear }\end{array}$ & $\begin{array}{l}\text { No. of } \\
\text { kernels/ row }(\operatorname{ard} / \text { fed })\end{array}$ \\
\hline Sakha & $65.18 \mathrm{a}$ & $287.96 \mathrm{a}$ & $162.10 \mathrm{a}$ & $23.86 \mathrm{a}$ & $5.00 \mathrm{a}$ & $15.70 \mathrm{a}$ & $46.36 \mathrm{a}$ & $38.77 \mathrm{a}$ \\
Gemmeiza & $64.17 \mathrm{~b}$ & $264.20 \mathrm{~b}$ & $143.75 \mathrm{~b}$ & $22.29 \mathrm{~b}$ & $4.70 \mathrm{~b}$ & $15.11 \mathrm{~b}$ & $44.35 \mathrm{a}$ & $31.27 \mathrm{~b}$ \\
Mallawy & $61.05 \mathrm{c}$ & $233.0 \mathrm{c}$ & $126.25 \mathrm{c}$ & $18.43 \mathrm{c}$ & $4.49 \mathrm{c}$ & $13.42 \mathrm{c}$ & $35.02 \mathrm{~b}$ & $28.10 \mathrm{c}$ \\
CV\% & 1.44 & 4.16 & 6.19 & 6.33 & 6.10 & 6.61 & 8.65 & 9.85 \\
\hline LSD 0.05 & 0.534 & 6.328 & 4.358 & 0.712 & 0.108 & 0.461 & 3.095 & 1.518 \\
LSD 0.01 & 0.77 & 9.129 & 6.286 & 1.028 & 0.156 & 0.664 & 4.464 & 2.189 \\
\hline
\end{tabular}

Mean performance of 28 hybrids and two checks for eight traits across three locations are shown in Table (3). The earlier hybrids were $\mathrm{P}_{2} \times \mathrm{P}_{3}$, SC128 and $\mathrm{P}_{3} \times \mathrm{P}_{5}$, while the highest hybrids for plant and ear heights were $\mathrm{P}_{3} \times \mathrm{P}_{4}, \mathrm{SC} 10$ and $\mathrm{P}_{2} \times \mathrm{P}_{4}$. Meanwhile, the shortest hybrids was $\mathrm{P}_{7} \times \mathrm{P}_{8}$ for plant height and $\mathrm{P}_{5} \times \mathrm{P}_{8}$ for ear height. Also, the highest hybrids were $\mathrm{P}_{3} \times \mathrm{P}_{4}, \mathrm{P}_{5} \times \mathrm{P}_{7}, \mathrm{P}_{5} \times \mathrm{P}_{6}$ and $\mathrm{P}_{4} \times \mathrm{P}_{5}$ for ear length, $\mathrm{P}_{2} \times \mathrm{P}_{5}$ and $\mathrm{P}_{3} \times \mathrm{P}_{6}$ for ear diameter, $\mathrm{P}_{1} \times$ $\mathrm{P}_{7}$ and $\mathrm{P}_{1} \times \mathrm{P}_{6}$ for number of rows/ear, $\mathrm{P}_{5} \times \mathrm{P}_{6}, \mathrm{P}_{5} \mathrm{x}$ $\mathrm{P}_{7}$ and $\mathrm{P}_{3} \times \mathrm{P}_{4}$ for number of kernels/row. For grain yield, the hybrids $\mathrm{P}_{4} \times \mathrm{P}_{7}, \mathrm{P}_{4} \times \mathrm{P}_{5}, \mathrm{P}_{5} \times \mathrm{P}_{7}$ and $\mathrm{P}_{6} \times \mathrm{P}_{7}$ gave the highest grain yield (over $>36$ ard/fed). Moreover, they were not significant out yield compared to two checks SC10 and SC128. These hybrids will be tested in yield trails for further evaluation.

Estimates of mean squares for general (GCA) and specific (SCA) combining ability and their interactions with locations are presented in Table (4). The mean squares values for GCA and SCA were significant for all studied traits except GCA for grain yield and SCA for ear height, ear diameter and number of kernels/row, indicating that both additive and non additive gene effects were involved in determining the performance of single progeny in most traits. The interaction mean squares due to GCA $x$ L and SCA $x$ L were significant for all traits except SCA x L for ear length, number of rows/ear and number of kernels/row, meaning that both 
additive and non-additive gene effects were affects by environment in most traits. These results agree with Nass et al. (2000), Mosa (2003), Glover et al. (2005), Bidhendi et al. (2012) and Umar et al. (2014)

Table 3. Mean performance of $28 \mathrm{~F}_{1}$ hybrids and two checks SC10 and SC128 for eight traits across locations

\begin{tabular}{|c|c|c|c|c|c|c|c|c|c|}
\hline$\overline{\text { Cros }}$ & & \multirow{2}{*}{$\begin{array}{c}\text { Days to } \\
50 \% \\
\text { silking } \\
63.16\end{array}$} & \multirow{2}{*}{$\begin{array}{c}\begin{array}{c}\text { Plant } \\
\text { height }(\mathrm{cm})\end{array} \\
269.58\end{array}$} & \multirow{2}{*}{$\begin{array}{c}\begin{array}{c}\text { Ear } \\
\text { height } \\
(\mathrm{cm})\end{array} \\
151.58\end{array}$} & \multirow{2}{*}{$\begin{array}{c}\begin{array}{c}\text { Ear } \\
\text { length } \\
(\mathrm{cm})\end{array} \\
19.31\end{array}$} & \multirow{2}{*}{$\begin{array}{c}\text { Ear } \\
\text { diameter } \\
(\mathrm{cm})\end{array}$} & \multirow{2}{*}{$\begin{array}{c}\begin{array}{c}\text { No. of } \\
\text { rows/ear }\end{array} \\
14.73\end{array}$} & \multirow{2}{*}{$\begin{array}{c}\begin{array}{c}\text { No. of } \\
\text { kernels/ row }\end{array} \\
38.15\end{array}$} & \multirow{2}{*}{$\begin{array}{c}\begin{array}{c}\text { Grain yield } \\
\text { (ard/fed) }\end{array} \\
32.77\end{array}$} \\
\hline $\mathrm{P}_{1} \mathrm{X}$ & $\mathrm{P}_{2}$ & & & & & & & & \\
\hline & $\mathrm{P}_{3}$ & 62.75 & 260.16 & 138.00 & 20.28 & 4.66 & 15.46 & 39.28 & 31.16 \\
\hline & $\mathrm{P}_{4}$ & 64.83 & 259.58 & 145.41 & 20.36 & 4.63 & 14.75 & 39.51 & 29.02 \\
\hline & $\mathrm{P}_{5}$ & 62.58 & 269.16 & 144.83 & 22.46 & 4.68 & 15.71 & 42.46 & 29.96 \\
\hline & $\mathrm{P}_{6}$ & 63.50 & 257.08 & 141.83 & 20.31 & 4.86 & 16.15 & 38.78 & 34.30 \\
\hline & $\mathrm{P}_{7}$ & 64.33 & 263.91 & 139.50 & 19.60 & 4.96 & 16.90 & 40.36 & 33.95 \\
\hline & $\mathrm{P}_{8}$ & 62.75 & 252.00 & 137.25 & 20.30 & 4.78 & 15.58 & 40.28 & 33.00 \\
\hline \multirow[t]{6}{*}{$\mathrm{P}_{2} \mathrm{x}$} & $\mathrm{P}_{3}$ & 60.41 & 260.41 & 141.66 & 20.96 & 4.56 & 13.48 & 40.28 & 30.47 \\
\hline & $\mathrm{P}_{4}$ & 65.16 & 278.16 & 157.08 & 20.21 & 4.60 & 14.35 & 37.85 & 34.16 \\
\hline & $\mathrm{P}_{5}$ & 63.91 & 268.50 & 154.41 & 22.26 & 5.01 & 13.78 & 44.63 & 32.74 \\
\hline & $\mathrm{P}_{6}$ & 63.16 & 266.25 & 152.91 & 22.26 & 4.81 & 14.50 & 42.86 & 35.10 \\
\hline & $\mathrm{P}_{7}$ & 63.08 & 253.00 & 146.08 & 20.06 & 4.68 & 14.58 & 39.71 & 27.62 \\
\hline & $\mathrm{P}_{8}$ & 63.25 & 259.75 & 148.33 & 21.61 & 4.91 & 14.08 & 41.36 & 34.87 \\
\hline \multirow[t]{5}{*}{$\mathrm{P}_{3} \mathrm{X}$} & $\mathrm{P}_{4}$ & 64.58 & 288.00 & 156.50 & 23.83 & 4.60 & 15.35 & 45.41 & 34.34 \\
\hline & $\mathrm{P}_{5}$ & 61.41 & 257.50 & 139.25 & 22.51 & 4.60 & 13.96 & 43.76 & 32.48 \\
\hline & $\mathrm{P}_{6}$ & 62.16 & 260.58 & 138.25 & 22.00 & 4.97 & 14.96 & 42.11 & 32.27 \\
\hline & $\mathrm{P}_{7}$ & 63.16 & 270.75 & 147.00 & 22.55 & 4.75 & 15.10 & 43.20 & 28.16 \\
\hline & $\mathrm{P}_{8}$ & 65.33 & 258.58 & 140.50 & 21.58 & 4.48 & 14.03 & 41.90 & 28.00 \\
\hline \multirow[t]{4}{*}{$\mathrm{P}_{4} \mathrm{X}$} & $\mathrm{P}_{5}$ & 64.91 & 267.58 & 143.33 & 23.28 & 4.58 & 14.28 & 45.10 & 36.53 \\
\hline & $\mathrm{P}_{6}$ & 65.66 & 259.33 & 140.16 & 20.06 & 4.50 & 14.01 & 38.70 & 27.62 \\
\hline & $\mathrm{P}_{7}$ & 64.58 & 262.75 & 145.50 & 21.56 & 4.63 & 15.43 & 42.98 & 37.37 \\
\hline & $\mathrm{P}_{8}$ & 64.83 & 264.08 & 152.16 & 21.53 & 4.68 & 14.53 & 40.76 & 31.80 \\
\hline \multirow[t]{3}{*}{$\mathrm{P}_{5} \mathrm{X}$} & $\mathrm{P}_{6}$ & 62.83 & 252.17 & 138.25 & 23.35 & 4.80 & 13.93 & 46.10 & 35.19 \\
\hline & $\mathrm{P}_{7}$ & 63.33 & 266.08 & 146.66 & 23.70 & 4.90 & 15.31 & 46.06 & 36.31 \\
\hline & $\mathrm{P}_{8}$ & 62.83 & 243.50 & 131.91 & 22.16 & 4.73 & 14.25 & 44.20 & 32.56 \\
\hline \multirow[t]{2}{*}{$\mathrm{P}_{6} \mathrm{X}$} & $\mathrm{P}_{7}$ & 63.41 & 247.83 & 136.91 & 21.88 & 4.93 & 15.60 & 41.93 & 36.07 \\
\hline & $\mathrm{P}_{8}$ & 64.25 & 255.33 & 139.83 & 21.03 & 4.71 & 15.03 & 42.61 & 27.69 \\
\hline & $\mathrm{P}_{8}$ & 62.83 & 240.25 & 132.83 & 21.83 & 4.83 & 15.21 & 42.73 & 34.43 \\
\hline \multicolumn{2}{|c|}{ SC-10 } & 63.92 & 279.17 & 156.08 & 21.37 & 4.48 & 13.57 & 42.43 & 36.06 \\
\hline \multicolumn{2}{|c|}{ SC-128 } & 61.17 & 260.75 & 137.00 & 21.60 & 4.78 & 14.06 & 41.77 & 35.38 \\
\hline \multicolumn{2}{|c|}{ LSD 0.05} & 1.46 & 14.18 & 12.45 & 1.57 & 0.322 & 1.14 & 3.81 & 5.53 \\
\hline \multicolumn{2}{|c|}{ LSD 0.01} & 1.91 & 18.57 & 16.30 & 2.06 & 0.422 & 1.50 & 4.99 & 7.25 \\
\hline
\end{tabular}

Table 4. Estimates of mean squares for general and specific combining ability and their interactions with locations.

\begin{tabular}{lccccccccc}
\hline SOV & df & $\begin{array}{c}\text { Days to } \\
50 \% \\
\text { silking }\end{array}$ & $\begin{array}{c}\text { Plant } \\
\text { height }\end{array}$ & $\begin{array}{c}\text { Ear } \\
\text { height }\end{array}$ & Ear length & $\begin{array}{c}\text { Ear } \\
\text { diameter }\end{array}$ & $\begin{array}{c}\text { No. of } \\
\text { rows/ear }\end{array}$ & $\begin{array}{c}\text { No. of } \\
\text { kernels/ } \\
\text { row }\end{array}$ & $\begin{array}{c}\text { Grain } \\
\text { yield }\end{array}$ \\
\hline GCA & 7 & $39.6^{* *}$ & $2460.03^{* *}$ & $1258.47^{*}$ & $49.284^{* *}$ & $0.504^{*}$ & $22.44^{* *}$ & $192.0^{* *}$ & 77.68 \\
SCA & 20 & $10.764^{* *}$ & $722.35^{* *}$ & 309.24 & $8.148^{* *}$ & 0.168 & $2.34^{* *}$ & 26.436 & $115.09^{* *}$ \\
GCA x L & 14 & $5.37^{* *}$ & $452.06^{* *}$ & $351.73^{* *}$ & $7.3^{* *}$ & $0.152^{*}$ & $4.97^{* *}$ & $25.71^{*}$ & $38.01^{* *}$ \\
SCA x L & 40 & $2.43^{* *}$ & $249.00^{* *}$ & $191.02^{* *}$ & 2.46 & $0.16^{* *}$ & 0.9 & 20.46 & $48.59^{* *}$ \\
\hline Error & 243 & 0.85 & 121.984 & 83.447 & 1.8 & 0.085 & 1.11 & 14.43 & 10.43 \\
\hline
\end{tabular}

*. ** Significant at 0.05 and 0.01 levels of probability, respectively

The improvement of maize yield depends on the knowledge of type of gene action involved in its inheritance and also the genetic control of related traits so the results in Table (5) exhibited magnitude of additive gene effects ( $\left.\mathrm{K}^{2} \mathrm{GCA}\right)$ higher than nonadditive gene effects $\left(\mathrm{K}^{2} \mathrm{SCA}\right)$ for ear height, ear length, ear diameter, number of rows/ear and numbers of kernels/row, indicating that the additive gene effect was most responsible for controlling inheritance of these trait. While, the non-additive gene effects was higher, than additive gene effects for days to $50 \%$ silking, plant height and grain yield, meaning that important of additive gene effects in the inheritance of these traits. On the other side the 
interaction between the non-additive gene effects with locations $\left(\sigma^{2} \mathrm{SCA} \times \mathrm{L}\right)$ was higher than interaction between additive gene effects with locations $\left(\sigma^{2} \mathrm{GCA} \times \mathrm{L}\right)$ in most traits, indicating that the non-additive gene effects was more interacted with the environment, than additive gene effects. These results support the findings of Kalla et al. (2001), Medici et al. (2004), Mosa (2005), Machado et al. (2009), Gichuru et al. (2011) Shahrokhi et al. (2013) and Mosa et al. (2015).

Table 5. Estimates of genetic components and their interaction with locations.

\begin{tabular}{|c|c|c|c|c|c|c|c|c|}
\hline Genetic parameters & $\begin{array}{c}\text { Days to } 50 \% \\
\text { silking }\end{array}$ & $\begin{array}{l}\text { Plant } \\
\text { height }\end{array}$ & $\begin{array}{l}\text { Ear } \\
\text { height }\end{array}$ & $\begin{array}{l}\text { Ear } \\
\text { length }\end{array}$ & $\begin{array}{l}\text { Ear } \\
\text { diameter }\end{array}$ & $\begin{array}{l}\text { No. of } \\
\text { ear }\end{array}$ & $\begin{array}{c}\text { rows/No. of } \\
\text { row }\end{array}$ & $\begin{array}{c}\text { kernels/Grain } \\
\text { yield }\end{array}$ \\
\hline $\mathrm{K}^{2} \mathrm{GCA}$ & 0.47 & 27.88 & 12.59 & 0.580 & 0.004 & 0.242 & 2.300 & 0.550 \\
\hline $\mathrm{K}^{2} \mathrm{SCA}$ & 0.69 & 39.44 & 9.85 & 0.474 & 0.0006 & 0.120 & 0.498 & 5.540 \\
\hline$\sigma^{2} \mathrm{GCA} \times \mathrm{L}$ & 0.188 & 13.75 & 11.17 & 0.229 & 0.002 & 0.161 & 0.470 & 1.140 \\
\hline$\sigma^{2} \mathrm{SCA} \times \mathrm{L}$ & 0.395 & 31.75 & 26.89 & 0.165 & 0.018 & 0.000 & 1.500 & 9.540 \\
\hline
\end{tabular}

Estimates of general combining ability effects of each parental lines for eight traits are presented in Table (6). The best general combiner inbred lines were $\mathrm{P}_{3}$ and $\mathrm{P}_{5}$ for earliness, $\mathrm{P}_{8}$ for short plant and ear heights, $\mathrm{P}_{5}$ for ear length number of kernels/row and grain yield, $\mathrm{P}_{7}$ for ear diameter and $\mathrm{P}_{1}$ and $\mathrm{P}_{7}$ for number of rows/ear. Malik et al. (2004) stated that the lines which had higher GCA effects can be used in synthetic variety development more effectively.

Table 6. Estimates of general combining ability (GCA) effects of each parental lines for eight traits across locations

\begin{tabular}{lllllllll}
\hline Inbred & $\begin{array}{l}\text { Days to } \\
50 \% \\
\text { silking }\end{array}$ & $\begin{array}{l}\text { Plant } \\
\text { height }\end{array}$ & $\begin{array}{l}\text { Ear } \\
\text { height }\end{array}$ & $\begin{array}{l}\text { Ear } \\
\text { length }\end{array}$ & $\begin{array}{l}\text { Ear } \\
\text { diameter }\end{array}$ & $\begin{array}{l}\text { No. of } \\
\text { rows/ear }\end{array}$ & $\begin{array}{l}\text { No. of } \\
\text { kernels/ row }\end{array}$ & $\begin{array}{l}\text { Grain } \\
\text { yield }\end{array}$ \\
\hline $\mathrm{P}_{1}$ & -0.142 & 0.586 & -1.430 & $-1.347^{* *}$ & 0.037 & $0.920^{* *}$ & $-2.406^{* *}$ & -0.554 \\
$\mathrm{P}_{2}$ & -0.434 & 4.614 & $7.513^{* *}$ & $-0.670^{*}$ & 0.037 & $-0.710^{*}$ & $-1.404^{*}$ & 0.042 \\
$\mathrm{P}_{3}$ & $-0.822^{* *}$ & 4.670 & -0.972 & 0.499 & -0.090 & -0.235 & 0.445 & $-1.768^{*}$ \\
$\mathrm{P}_{4}$ & $1.635^{* *}$ & $8.586^{* *}$ & $5.527^{*}$ & 0.021 & $-0.156^{* *}$ & -0.177 & -0.493 & 0.559 \\
$\mathrm{P}_{5}$ & $-0.50^{*}$ & -0.579 & -1.388 & $1.502^{* *}$ & 0.023 & -0.421 & $3.173 * *$ & $1.40^{*}$ \\
$\mathrm{P}_{6}$ & 0.038 & -4.899 & -3.138 & 0.029 & 0.068 & 0.070 & -0.029 & 0.124 \\
$\mathrm{P}_{7}$ & -0.003 & -3.899 & -2.083 & 0.077 & $0.092^{*}$ & $0.728^{*}$ & 0.618 & 1.072 \\
$\mathrm{P}_{8}$ & 0.218 & $-9.079 * *$ & $-4.027 *$ & -0.111 & -0.004 & -0.174 & 0.095 & -0.855 \\
\hline LSD g $_{\mathrm{i}} 0.05$ & 0.50 & 5.01 & 4.02 & 0.64 & 0.092 & 0.52 & 1.19 & 1.40 \\
LSD g g $_{\mathrm{i}} 0.01$ & 0.76 & 6.96 & 6.14 & 0.88 & 0.13 & 0.73 & 1.66 & 2.01 \\
\hline
\end{tabular}

*. ** Significant at 0.05 and 0.01 levels of probability, respectively

Estimates of the specific combining ability effects for 28 hybrids are presented in Table (7). The desirable hybrids for specific combining ability effects were $\mathrm{P}_{1} \times \mathrm{P}_{8}, \mathrm{P}_{2} \times \mathrm{P}_{3}, \mathrm{P}_{3} \times \mathrm{P}_{5}$ and $\mathrm{P}_{7} \times \mathrm{P}_{8}$ for earliness, $\mathrm{P}_{2} \times \mathrm{P}_{3}$ and $\mathrm{P}_{5} \times \mathrm{P}_{8}$ for short plant and ear heights and $\mathrm{P}_{3} \times \mathrm{P}_{4}$ and $\mathrm{P}_{5} \times \mathrm{P}_{7}$ for high plant and ear heights, $\mathrm{P}_{1} \times \mathrm{P}_{5}, \mathrm{P}_{2} \times \mathrm{P}_{6}, \mathrm{P}_{2} \times \mathrm{P}_{8}$ and $\mathrm{P}_{3} \times \mathrm{P}_{4}$ for ear length, $\mathrm{P}_{2} \times \mathrm{P}_{5}$ and $\mathrm{P}_{3} \times \mathrm{P}_{6}$ for ear diameter, $\mathrm{P}_{1} \times \mathrm{P}_{7}$,
$\mathrm{P}_{2} \times \mathrm{P}_{4}$ and $\mathrm{P}_{3} \times \mathrm{P}_{4}$ for number of rows/ear, $\mathrm{P}_{2} \times \mathrm{P}_{6}$ and $\mathrm{P}_{3} \times \mathrm{P}_{4}$ for number of kernels/row and $\mathrm{P}_{4} \times \mathrm{P}_{7}$ for grain yield. In general, the good specific combiners for different studied traits involved parents with high $\mathrm{x}$ high, high $\mathrm{x}$ low, low $\mathrm{x}$ high and low $\mathrm{x}$ low general combinations. Also, can be used above hybrids as a potential single crosses combinations and tested further evaluation.

Table 7. Specific combining ability effects of 28 hybrids for eight traits across locations

\begin{tabular}{|c|c|c|c|c|c|c|c|c|c|}
\hline \multicolumn{2}{|l|}{ Cross } & $\begin{array}{c}\text { Days to } \\
50 \% \text { silking }\end{array}$ & $\begin{array}{l}\text { Plant } \\
\text { height }\end{array}$ & $\begin{array}{c}\text { Ear } \\
\text { height }\end{array}$ & $\begin{array}{l}\text { Ear } \\
\text { length }\end{array}$ & $\begin{array}{c}\text { Ear } \\
\text { diameter }\end{array}$ & $\begin{array}{l}\text { No. of } \\
\text { rows/ear }\end{array}$ & $\begin{array}{c}\text { No. of } \\
\text { kernels/ } \\
\text { row }\end{array}$ & $\begin{array}{l}\text { Grain } \\
\text { yield }\end{array}$ \\
\hline $\mathrm{P}_{1} \mathrm{X}$ & $\mathrm{P}_{2}$ & 0.204 & 3.24 & 1.643 & -0.199 & -0.014 & -0.302 & 0.062 & 0.787 \\
\hline & $\mathrm{P}_{3}$ & 0.177 & -6.23 & -3.454 & -0.402 & -0.017 & -0.043 & -0.654 & 0.983 \\
\hline & $\mathrm{P}_{4}$ & -0.198 & $-10.73 * *$ & -2.538 & 0.159 & 0.013 & $-0.818^{* *}$ & 0.518 & $-3.481 *$ \\
\hline & $\mathrm{P}_{5}$ & -0.323 & $8.02 *$ & 3.796 & $0.779 *$ & -0.117 & 0.392 & -0.199 & $-3.4^{*}$ \\
\hline & $\mathrm{P}_{6}$ & 0.063 & 0.26 & 2.546 & 0.101 & 0.021 & 0.334 & -0.679 & 2.232 \\
\hline & $\mathrm{P}_{7}$ & $0.940 *$ & 6.09 & -0.843 & -0.663 & 0.102 & $0.425 *$ & 0.257 & 0.934 \\
\hline & $\mathrm{P}_{8}$ & $-0.865^{*}$ & -0.65 & -1.149 & 0.226 & 0.010 & 0.011 & 0.696 & 1.909 \\
\hline
\end{tabular}




\begin{tabular}{|c|c|c|c|c|c|c|c|c|c|}
\hline \multicolumn{10}{|c|}{ Table 7 Cont. } \\
\hline \multirow[t]{6}{*}{$\mathrm{P}_{2} \mathrm{x}$} & $\mathrm{P}_{3}$ & $-1.865 * *$ & $-10.01 *$ & $-8.732 *$ & -0.396 & -0.117 & -0.396 & -0.657 & -0.301 \\
\hline & $\mathrm{P}_{4}$ & 0.427 & 3.83 & 0.185 & -0.669 & -0.019 & $0.411 *$ & -2.152 & 1.060 \\
\hline & $\mathrm{P}_{5}$ & $1.302 * *$ & 3.33 & 4.436 & -0.099 & $0.216^{*}$ & 0.089 & 0.965 & -1.176 \\
\hline & $\mathrm{P}_{6}$ & 0.024 & 5.39 & 4.865 & $1.373 * *$ & -0.028 & 0.314 & $2.401 *$ & 2.432 \\
\hline & $\mathrm{P}_{7}$ & -0.018 & $-8.86^{*}$ & -3.204 & $-0.874 *$ & -0.181 & -0.26 & -1.396 & $-5.989 * *$ \\
\hline & $\mathrm{P}_{8}$ & -0.073 & 3.08 & 0.990 & $0.865^{*}$ & 0.144 & 0.142 & 0.776 & 3.187 \\
\hline \multirow[t]{5}{*}{$\mathrm{P}_{3} \mathrm{x}$} & $\mathrm{P}_{4}$ & 0.232 & $13.60 * *$ & $8.087 *$ & $1.779 * *$ & 0.110 & $0.936 * *$ & $3.564 * *$ & 3.046 \\
\hline & $\mathrm{P}_{5}$ & $-0.810 *$ & -7.73 & -2.246 & $-1.019 * *$ & -0.069 & -0.202 & -1.572 & 0.369 \\
\hline & $\mathrm{P}_{6}$ & -0.587 & -0.33 & -1.496 & -0.063 & $0.235^{*}$ & 0.306 & -0.199 & 1.417 \\
\hline & $\mathrm{P}_{7}$ & 0.454 & $8.84^{*}$ & 6.198 & 0.440 & 0.016 & -0.218 & 0.237 & $-3.637 *$ \\
\hline & $\mathrm{P}_{8}$ & $2.399 * *$ & 1.85 & 1.643 & -0.338 & -0.158 & -0.382 & -0.54 & -1.877 \\
\hline \multirow[t]{4}{*}{$\mathrm{P}_{4} \mathrm{X}$} & $\mathrm{P}_{5}$ & 0.232 & -1.56 & -4.663 & 0.226 & -0.022 & 0.056 & 0.521 & 2.089 \\
\hline & $\mathrm{P}_{6}$ & 0.454 & -5.49 & -6.079 & $-1.519 * *$ & -0.150 & $-0.702 * *$ & $-2.677 *$ & $-5.558 * *$ \\
\hline & $\mathrm{P}_{7}$ & -0.587 & -3.08 & -1.802 & -0.066 & -0.036 & 0.056 & 0.960 & $3.441 *$ \\
\hline & $\mathrm{P}_{8}$ & -0.560 & 3.44 & $6.81 *$ & 0.090 & 0.105 & 0.059 & -0.735 & -0.398 \\
\hline \multirow[t]{3}{*}{$\mathrm{P}_{5} \mathrm{x}$} & $\mathrm{P}_{6}$ & -0.254 & -3.49 & -1.079 & 0.284 & -0.031 & $-0.54 *$ & 1.057 & 1.184 \\
\hline & $\mathrm{P}_{7}$ & 0.288 & $9.42 *$ & $6.282 *$ & 0.587 & 0.049 & 0.184 & 0.376 & 1.362 \\
\hline & $\mathrm{P}_{8}$ & -0.435 & $-7.98 *$ & $-6.824 *$ & -0.758 & -0.025 & 0.02 & -0.968 & -0.463 \\
\hline \multirow[t]{2}{*}{$\mathrm{P}_{6} \mathrm{x}$} & $\mathrm{P}_{7}$ & -0.157 & -4.51 & -1.718 & 0.242 & 0.038 & -0.024 & -0.554 & 2.371 \\
\hline & $\mathrm{P}_{8}$ & 0.454 & $8.17 *$ & 3.143 & -0.419 & -0.086 & 0.311 & 0.651 & $-4.078 *$ \\
\hline $\mathrm{P}_{7} \mathrm{x}$ & $\mathrm{P}_{8}$ & $-0.921 *$ & $-7.91 *$ & -4.913 & 0.334 & 0.010 & -0.163 & 0.121 & 1.719 \\
\hline \multicolumn{2}{|c|}{ LSD $_{\mathrm{ij}} 0.05$} & 0.76 & 7.77 & 6.81 & 0.770 & 0.190 & 0.40 & 2.20 & 3.43 \\
\hline \multicolumn{2}{|c|}{$\operatorname{LSD~S}_{\mathrm{ij}} 0.01$} & 1.02 & 10.39 & 9.1 & 1.000 & 0.260 & 0.62 & 2.97 & 4.59 \\
\hline
\end{tabular}

Significant at 0.05 and 0.01 levels of probability, respectively.

\section{References}

Bidhendi, M.Z.; R. Choukan; F. Darvish; K. Mostafavi and E. Majidi (2012). Classification of maize inbred lines into heterotic groups using diallel analysis. International Journal Biological, Biomolecular, Agricultural, Food and Biotechnological Engineering, 6: 556-559.

Comstoc, R.E. and H.F. Moll (1963). Genotypeenvironmental interactions. National Academy of Science, National Research Council, Publication, 982: 164-196.

Frey, K.J. (1964). Adaptation reaction of oat strains selected under stress and non-stress environmental conditions. Crop Sci. 4: 55-58.

Gichuru, L.; K. Njoroge; J. Ininda and L. Peter (2011). Combining ability of grain yield and agronomic traits in diverse maize lines with maize streak virus resistance for eastern Africa region. Agric. Biol. J. N. Am. 2: 432-439.

Glover, Max A.; D.B. Willmot; L.L. Darrah; B. E. Hibbard and X. Zhu (2005). Diallel analysis of agronomic traits using Chinese and U.S. Maize germplasm. Crop Sci. 45: 1096-1102.

Griffing, B. (1956). Concepts of general and specific combining ability in relation to diallel crossing systems. Aust. J. Biological Sci. 9: 463-493.

Kalla, V.; R. Kumar and A.K. Basandrai (2001). Combining ability analysis and gene action estimates of yield and yield contributing characters in maize. Crop Res. Hisar 22: 102-106.

Machado, J.C.; J.C. Souza; M.A. Ramalho and J.L. Lima (2009). Stability of combining ability effects in maize hybrids. Scien. Agric. 66: 494498.

Malik, S.I.; H.N. Malik; N.M. Minhas and M. Munir (2004). General and specific combining ability studies in maize diallel crosses. Int. J. Agric. Biol. 6: 856-859.

Medici, L.O.; M.B. Pereira; P.J. Lea and R.A. Azevedo (2004). Diallel analysis of maize lines with contrasting responses to applied nitrogen. J. Agric. Sci. 142: 535-541.

Mosa, H.E. (2003). Heterosis and combining ability in maize (Zea mays L.). Minufiya J. Agric. Res. 28: $1375-1386$.

Mosa, H.E. (2005). Combining ability of eight yellow maize (Zea mays L.) inbred lines for different characters in diallel crosses. J. Agric. Res. Tanta Univ. 31: 604-615.

Mosa,H.E.; M.A.A.Hassan and S.M.Abo ElHaress (2015). Combining ability for new maize inbred lines for grain yield and some agronomic traits. Minufiya J.Agric.Res. 40:109-117.

Nass, L.L.; M. Lima; R. Vencovsky and P.B. Gallo (2000). Combining ability of maize inbred lines evaluated in three environments in Brazil. Science and Agriculture 57: 129-134.

Rojas, B.A. and G.F. Sprague (1952). A comparison of variance in corn yield trails. III. General and specific combining ability and their interaction with locations and years. Agron. J. 44: 462-466.

Shahrokhi, M.; S.K. Khorasani and A. Ebrahimi (2013). Study of genetic components in various maize (Zea mays L.) traits using generation mean 
analysis method, International Journal of Agronomy and Plant Production, 4:405-412.

Steel, R.G.D. and J.H. Torrie (1980). Principles and Procedures of Statistics. A Biometrical Approach, $2^{\text {nd }}$ Ed. Mc Graw Hill, N.Y. USA.

Umar, U.U.; S.G.Ado ; D. A. Aba and S.M.

Bugaje (2014). Estimates of combining ability and gene action in maize(Zea mays L.) under water stress and non-stress conditions. Journal of Biology, Agriculture and Healthcare, 4:247-254.

Yan, W. and A. Hunt (2003). Biplot analysis of diallel data. Crop Sci. 42: 21-30.

\title{
تحليل القدرة على الانتلاف وطبيعة الفعل الجينى للمحصول ومكوناته لبعض سلالات الذرة الشامية البيضاء
}

\author{
حاتم الحمادى موسى ، إبراهيم عبد التبى إبراهيم الجزار ، محمد عرفة على حسن

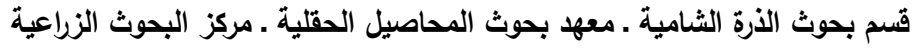

\begin{abstract}
تم تحليل القدرة على الائتلاف لصفة المحصول ومكوناته وارتفاع النبات والكوز وتاريخ خروج 50\% من حرائر النورات المؤنثة لـ 8

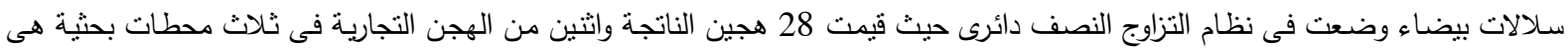

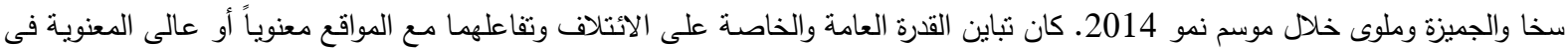
معظم الصفات ومع ذلك تأثنرات الفعل الوراثى المضيف أكثر تحكماً فى صفات ارتفاع الكوز وطول الكوز وقطر الكوز وعدد الصفوف بالكوز وعدد الحبوب بالصف بينما تأثيرات الفعل الوراثى غير المضيف هى الأكثر أهمية فى صفات تاريخ ظهور 50\% من حرائر النورات المؤنثة

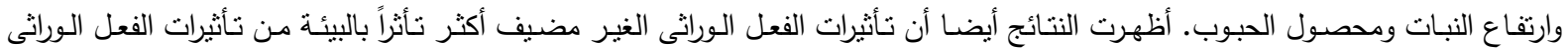

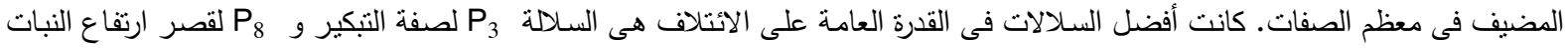
والكوز و P لزيادة طول الكوز وعدد الحبوب بالصف ومحصول الحبوب و P لقطر الكوز و P P لعدد الصفوف بالكوز . أفضل هجين فى القدرة

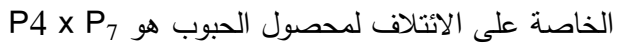

